\title{
Plasma proenkephalin A 119-159 on intensive care unit admission is a predictor of organ failure and 30-day mortality
}

\author{
Attila Frigyesi ${ }^{1,2^{*}} \mathbb{( D}$, Lisa Boström ${ }^{1,2}$, Maria Lengquist ${ }^{1,2}$, Patrik Johnsson ${ }^{1,3}$, Oscar H. M. Lundberg ${ }^{1,3}$, \\ Martin Spångfors ${ }^{1,4}$, Martin Annborn ${ }^{1,5}$, Tobias Cronberg ${ }^{1,6}$, Niklas Nielsen ${ }^{1,5}$, Helena Levin ${ }^{1,7}$ and Hans Friberg ${ }^{1,3}$
}

*Correspondence: attila.frigyesi@med.lu.se ${ }^{1}$ Department of Clinical Sciences, Anaesthesiology and Intensive Care, Lund University, 22185 Lund, Sweden

Full list of author information is available at the end of the article

\begin{abstract}
Background: Proenkephalin A 119-159 (penKid) has been suggested as a marker of renal failure and poor outcome. We aimed to investigate the association of penKid on ICU admission with organ dysfunction and mortality in a mixed ICU population. In this retrospective, observational study, admission penKid levels from prospectively collected blood samples of consecutive patients admitted to four Swedish ICUs were analysed. The association of penKid with day-two sequential organ failure assessment (SOFA) scores and 30-day mortality was investigated using (ordinal) logistic regression. The predictive power of penKid for 30-day mortality and dialysis was assessed using the area under the receiver operating characteristic curve (AUC).
\end{abstract}

Results: Of 1978 included patients, 632 fulfilled the sepsis 3-criteria, 190 had a cardiac arrest, and 157 had experienced trauma. Admission penKid was positively associated with 30-day mortality with an odds ratio of 1.95 (95\% confidence interval 1.75-2.18, p< 0.001 ), and predicted 30-day mortality in the entire ICU population with an AUC of 0.71 (95\% confidence interval 0.68-0.73) as well as in the sepsis, cardiac arrest and trauma subgroups (AUCs of 0.61-0.84). Correction for admission plasma creatinine revealed that penKid correlated with neurological dysfunction.

Conclusion: Plasma penKid on ICU admission is associated with day-two organ dysfunction and predictive of 30-day mortality in a mixed ICU-population, as well as in sepsis, cardiac arrest and trauma subgroups. In addition to being a marker of renal dysfunction, plasma penKid is associated with neurologic dysfunction in the entire ICU population, and cardiovascular dysfunction in sepsis.

Keywords: Proenkephalin, Biomarker, Intensive care, Mortality, Organ dysfunction

\section{Introduction}

The severity of illness on ICU admission is commonly assessed using the Simplified Acute Physiology Score III (SAPS-3), which includes laboratory measures, physiological parameters, and comorbidities $[1,2]$. There is a need to improve and simplify the assessment of the severity of disease and if possible characterise disease phenotypes, e.g. using biomarkers. party material in this article are included in the article's Creative Commons licence, unless indicated otherwise in a credit line to the material. If material is not included in the article's Creative Commons licence and your intended use is not permitted by statutory regulation or exceeds the permitted use, you will need to obtain permission directly from the copyright holder. To view a copy of this licence, visit http:// creativecommons.org/licenses/by/4.0/. 
Proenkephalin A 119-159 (penKid) is a peptide derived from the same precursor as met- and leu-enkephalins and it has been established as a reliable surrogate marker for the unstable enkephalins. It is stable in plasma for at least $48 \mathrm{~h}$ [3]. The gene for penKid is expressed in the central nervous system and in multiple non-neuronal tissues [4], with concentrations of penKid being 100-fold higher in the cerebrospinal fluid compared with serum [3].

Serum penKid has been suggested to be a biomarker for assessment of renal function [5, 6], and an earlier marker of acute kidney injury (AKI) than creatinine [7]. Dépret and colleagues showed in a cohort of critically ill patients that subclinical AKI, defined by elevated penKid $>80 \mathrm{pmol} / \mathrm{L}$ in patients without AKI, was associated with mortality similar to that of patients with AKI [8].

Proenkephalin A 119-159 is a small molecule assumed to be freely filtered through the renal glomerulus $[9,10]$ and does not seem to be directly affected by an inflammatory response, making penKid a specific marker for renal function in inflammatory states such as sepsis $[7,10,11]$. In addition to being a marker of renal function, serum penKid has been proposed to be a marker of brain injury. Yalcin et al. have shown that serum proenkephalin may be used as a predictor of mortality in patients with traumatic brain injury [12]. Associations between elevated penKid levels and functional outcome as well as mortality in patients with traumatic brain injury, ischaemic- and hemorrhagic stroke have also been found [13-16]. A combination of renal dysfunction and brain injury that might explain penKid elevation in critically ill patients has not been explored.

Our hypothesis was that penKid may be valuable for assessment of subsequent organ dysfunction and mortality in critically ill patients. We aimed to investigate the association and prognostic value of admission penKid with organ dysfunction and 30-day mortality in the ICU.

\section{Materials and methods} Study design and setting

The study design is a multicenter retrospective analysis and observational study of prospectively collected blood samples. The Standards for Reporting of Diagnostic Accuracy Studies (STARD) guidelines were followed [17].

\section{Participants}

Consecutive patients admitted to any of four mixed surgical and medical ICUs in southern Sweden in 2016 were evaluated for eligibility and in this group all adult patients with valid blood samples were included in the study. The sepsis subgroup was identified using the sepsis-3 criteria. A detailed description of this procedure has been presented elsewhere [18]. The cardiac arrest subgroup was identified using the International Statistical Classification of Diseases and Related Health Problems (ICD) code I46.9 at ICU discharge. The trauma subgroup was identified through polytrauma as the reason for ICU admission. When transfers occurred between the participating ICUs, follow-up data were merged to form cohesive ICU admissions. 


\section{Variables}

The third version of the Simplified Acute Physiology Score (SAPS-3) was calculated $[1,2]$ based on physiological parameters and laboratory findings recorded within $1 \mathrm{~h}$ before/after ICU admission.

The Sequential Organ Failure Assessment (SOFA) score was recorded daily during the ICU stay [19]. The day-two SOFA was the first score to be based on a full 24-h period following admission. The total SOFA score was based on the available SOFA subscores.

\section{Data sources}

Background and survival data were extracted from the Patient Administrative System for Intensive Care Units (PASIVA). PASIVA is the portal by which the treating physician and nursing staff submit prospectively collected laboratory and physiological data to the Swedish Intensive Care Registry (SIR). PASIVA is synchronised with the Swedish population register, which contains survival data.

The blood samples were collected in EDTA (ethylenediaminetetraacetic acid) vacutainers at ICU admission, centrifuged to obtain EDTA plasma, aliquoted, frozen, and thereafter stored in the SWECRIT biobank at Region Skåne (registration no. BD-47). For inclusion, the sample had to be collected within $6 \mathrm{~h}$ after ICU admission. If the sampling time was missing, samples were included if the time of freezing was within the 6-h time frame. The frozen plasma samples were shipped, and batch analysis of penKid was performed in a blinded fashion on thawed samples using a luminescence immunoassay technique [5] in 2019 at the laboratory of SphingoTec GmbH (Hennigsdorf, Germany). The assay has a limit of detection at $7 \mathrm{pmol} / \mathrm{L}$ and a mean interassay coefficient of variation of $5.7 \%$ in the measuring range $10.9-686 \mathrm{pmol} / \mathrm{L}$.

No clinical information was available to the performers/readers of the index test. The penKid results were not available to clinicians during patient care.

\section{Bias}

Selection bias was investigated in a comparison of baseline characteristics between included and excluded patients.

\section{Study size}

The study size was based on the number of ICU admissions and valid blood samples during the study period.

\section{Quantitative variables}

In this study, renal failure was defined as a renal SOFA score of $\geq 1$ for the purpose of subgroup analyses and $\geq 2$ when renal failure was treated as an outcome (AUC).

Reference levels for penKid have been published by Marino et al. 2015 [10] with a mean ( \pm 1 standard deviation) of $47 \pm 14 \mathrm{pmol} / \mathrm{L}$, and a 99 th percentile of $80 \mathrm{pmol} / \mathrm{L}$.

\section{Statistical methods}

The association between 30-day mortality and penKid levels was analysed using logistic regression. Plasma penKid was included as a base-10 log-transformed $\mathrm{z}$-normalised (linear transformation to 0 mean and standard deviation 1) independent variable. The 
regression models were evaluated with the Hosmer-Lemeshow goodness-of-fit test with ten groups, and only models resulting in non-significant tests were reported [20]. The association between day-two SOFA scores and penKid levels were analysed using multivariable ordinal logistic regression [21].

To evaluate the additional value of penKid to SAPS-3 in the logistic regression model, the area under the receiver operating characteristic curve (AUC) was calculated [22]. Differences in AUCs were tested with the method of DeLong et al. [23].

By adding admission creatinine as an independent variable in the ordinal logistic regression models, we examined the association of penKid with organ dysfunction independently of renal dysfunction.

\section{Results}

\section{Participants}

Out of 2546 adult ICU admissions, 1978 (78\%) had valid blood samples at ICU admission and did not opt out. Of the 1978 admissions, the sepsis subgroup constituted 32\% $(\mathrm{n}=632)$, the cardiac arrest subgroup 9.6\% $(\mathrm{n}=190)$ and the trauma subgroup $7.9 \%(\mathrm{n}$ $=157$ ). There was no overlap between the sepsis and the cardiac arrest subgroups (cardiac arrest was an exclusion criterion for sepsis). Of the trauma group $6.5 \%$ were identified as having sepsis 3 on admission, and $1.3 \%$ of the trauma patients also had a cardiac arrest. See Additional file 1: Figure S1 for a flow chart.

The median time from ICU admission to blood sampling for penKid was $21 \mathrm{~min}$ (interquartile range (IQR) $15-40 \mathrm{~min}$ ). The mean time from ICU admission to the start of day-two SOFA was $17.5 \mathrm{~h}$ (standard deviation $6.2 \mathrm{~h}$ ).

\section{Descriptive data}

The median (IQR) penKid concentration was 85 (54-148) pmol/L for the entire ICU population, 103 (63-194) pmol/L for the sepsis group, 136 (91-215) pmol/L in the cardiac arrest group, and 74 (52-103) pmol/L in the trauma group (Table 1). For the distribution of penKid, see Additional file 1: Figure S2. Fifty-three percent of the ICU patients were above the normal-range 99th percentile of $80 \mathrm{pmol} / \mathrm{L}$.

The female to male ratio, ICU length of stay, ICU mortality, 30-day mortality, morbidity as measured by SAPS-3, median age and variables included in the SAPS-3 score are presented in Table 1.

The patients excluded from the study were slightly younger, had lower mortality, and a shorter ICU length of stay, as presented in Additional file 1: Table S1. The excluded group included fewer sepsis patients but more trauma.

The fraction of missing parameters was mostly low, in the range of $0 \%$ for GCS to bilirubin and platelet counts of 7\% (Additional file 1: Table S2). Oxygenation parameters on admission were missing from $12 \%$ in $\mathrm{FiO}_{2}$ to $49 \%$ for $\mathrm{PaO}_{2}$. Day-two SOFA sub-scores were not available in the range of 49 to $53 \%$, almost entirely due to a short ICU length of stay.

\section{Unadjusted models}

Thirty-day mortality and day-two SOFA was higher for high penKid levels as presented in Fig. 1 and Additional file 1: Figure S3. 
Table 1 Descriptive statistics for the whole ICU population, the sepsis-3 subgroup, the cardiac arrest subgroup and the trauma subgroup

\begin{tabular}{|c|c|c|c|c|}
\hline & ICU & Sepsis & Cardiac arrest & Trauma \\
\hline Number of patients & 1978 & $632(32 \%)$ & $190(9.6 \%)$ & $157(7.9 \%)$ \\
\hline Women (\%) & 39 & 40 & 27 & 23 \\
\hline ICU length of stay (days) & $1.7(0.8-3.8)$ & $2.5(1.1-5.5)$ & $2.4(0.97-4.3)$ & $1.8(0.93-4.1)$ \\
\hline ICU mortality (\%) & 11 & 14 & 34 & 4.5 \\
\hline 30-day mortality (\%) & 22 & 28 & 54 & 12 \\
\hline SAPS-3 score & $59(47-71)$ & $66(57-77)$ & $76(66-87)$ & $48(39-59)$ \\
\hline Day-one SOFA score & $7(4-10)$ & $8(6-11)$ & $10(8-13)$ & $5(2-7)$ \\
\hline Day-two SOFA score $(n=995)$ & $8(5-10)$ & $8(6-11)$ & $10(8-12)$ & $6(4-9)$ \\
\hline CRRT during ICU stay (\%) & 9.2 & 15 & 7.4 & 0.6 \\
\hline \multicolumn{5}{|l|}{ Box I } \\
\hline Age (years) & $66(54-74)$ & $69(61-76)$ & $68(60-76)$ & $55(33-70)$ \\
\hline \multicolumn{5}{|l|}{ Comorbidities } \\
\hline Cancer therapy (\%) & 4.6 & 6.3 & 4.2 & 0.6 \\
\hline Chronic heart failure (\%) & 5.5 & 7.0 & 10 & 1.3 \\
\hline Hematological cancer (\%) & 2.6 & 5.2 & 1.0 & 0 \\
\hline Liver cirrhosis (\%) & 1.1 & 1.6 & 0 & 0 \\
\hline Cancer (\%) & 10.4 & 10.4 & 7.9 & 1.3 \\
\hline Vasoactive drugs before ICU (\%) & 44 & 47 & 64 & 21 \\
\hline \multicolumn{5}{|l|}{ Box II } \\
\hline \multicolumn{5}{|l|}{ Surgical status at ICU admission } \\
\hline No surgery (\%) & 74 & 85 & 94 & 83 \\
\hline \multicolumn{5}{|l|}{ Box III } \\
\hline GCS & $13(10-15)$ & $13(10-15)$ & $3(3-8)$ & $10(6-15)$ \\
\hline Total bilirubin $(\mu \mathrm{mol} / \mathrm{L})$ & $9(6-15)$ & $10(7-19)$ & $8(5-12)$ & $8(5-12)$ \\
\hline Max. temperature $\left({ }^{\circ} \mathrm{C}\right)$ & $37.0(36.3-37.6)$ & $37.3(36.5-38.1)$ & $36.0(35.5-36.8)$ & $37.0(36.0-37.5)$ \\
\hline Max. creatinine ( $\mu \mathrm{mol} / \mathrm{L})$ & $93(70-145)$ & $119(79-205)$ & $112(87-149)$ & $87(74-111)$ \\
\hline Max. heart rate (bpm) & $100(80-118)$ & $107(90-122)$ & $98(80-115)$ & $90(80-110)$ \\
\hline Max. leukocyte count $\left(\times 10^{9} / \mathrm{L}\right)$ & $13(8.8-18)$ & $13(8.4-19)$ & $16(12-21)$ & $14(11-19)$ \\
\hline Min. pH & $7.34(7.24-7.41)$ & $7.32(7.20-7.40)$ & $7.19(7.04-7.3)$ & $7.34(7.29-7.40)$ \\
\hline Min. platelet count $\left(\times 10^{9} / \mathrm{L}\right)$ & $221(161-286)$ & $218(147-304)$ & $222(165-270)$ & $212(171-261)$ \\
\hline Min. systolic blood pressure $(\mathrm{mmHg})$ & $100(80-120)$ & $91(75-115)$ & $85(68-109)$ & $104(90-130)$ \\
\hline \multicolumn{5}{|l|}{ Oxygenation } \\
\hline Initial respiratory support ${ }^{\dagger}(\%)$ & 58 & 59 & 86 & 53 \\
\hline $\mathrm{FiO}_{2}(\%)$ & $50(40-70)$ & $60(40-80)$ & $60(50-80)$ & $45(40-57)$ \\
\hline $\mathrm{PaO}_{2}(\mathrm{kPa})$ & $13(9.8-18)$ & $11(9.0-15)$ & $13(11-22)$ & $16(11-24)$ \\
\hline \multicolumn{5}{|l|}{ Other } \\
\hline penKid (pmol/L) & $85(54-148)$ & $103(63-194)$ & $136(91-215)$ & $74(52-103)$ \\
\hline
\end{tabular}

If not stated otherwise, values represent medians (interquartile ranges, IQR). Box I-III refers to the subsections of the SAPS-3 scoring system. TRespiratory support refers to invasive or non-invasive ventilation within $1 \mathrm{~h}$ of admission

ICU: intensive care unit, SAPS-3: simplified acute physiology score III, SOFA: Sequential Organ Failure Assessment, CRRT: continuous renal replacement therapy, GCS: Glasgow coma scale, $\mathrm{FiO}_{2}$ : fraction of inspired oxygen, $\mathrm{PaO}_{2}$ : arterial partial pressure of oxygen, penKid: Proenkephalin A 119-159

Unadjusted logistic regression models for 30-day mortality and day-two SOFA are presented in Table 2 and Fig. 3. Thirty-day mortality was higher for high penKid levels, with an odds ratio (OR) of 1.95 (95\% CI 1.74-2.18, $p<0.001)$ in the entire ICU population, 1.39 (95\% CI 1.18-1.64, $p<0.001)$ in the sepsis subgroup, 2.42 (95\% CI 


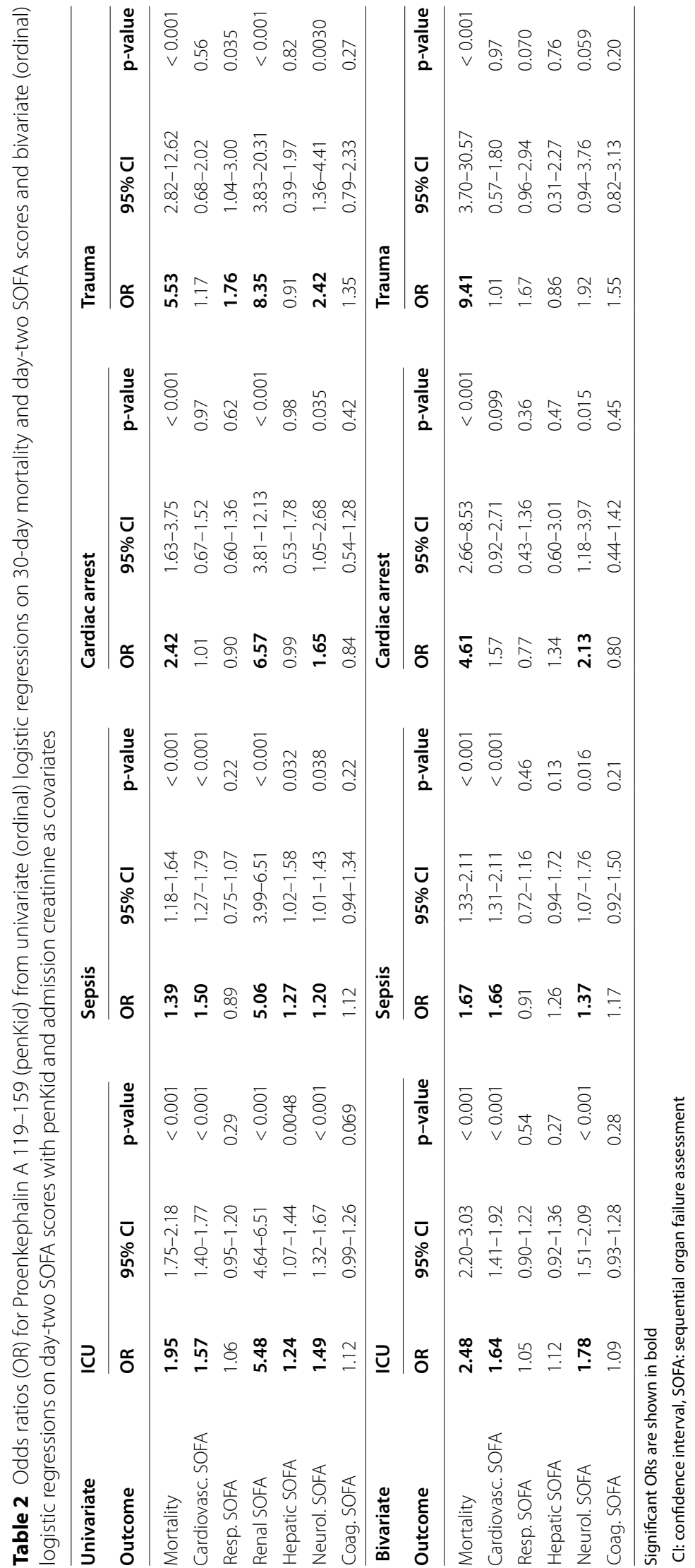




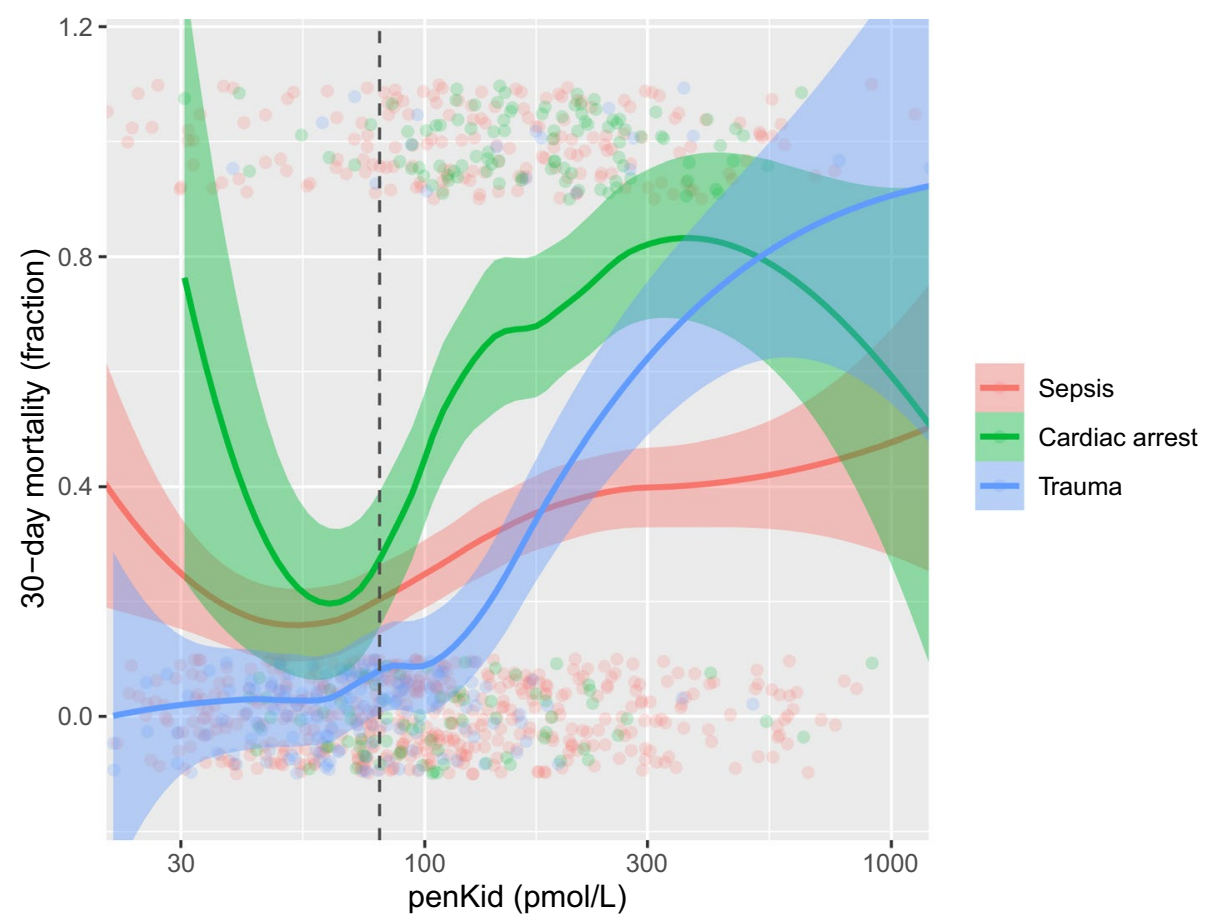

Fig. 1 Thirty-day mortality as a function of admission plasma proenkephalin A 119-159 (penKid) concentration in the subgroups sepsis, cardiac arrest and trauma. The vertical line at penKid $80 \mathrm{pmol} / \mathrm{L}$ is the upper normal (99th percentile) limit. The continuous lines represent smoothed averages, i.e. the average 30-day mortality in a small neighbourhood of a penKid value. The coloured bands around the smoothed averages indicate $95 \%$ confidence bands. Individual patients are represented by dots with jitter added. Thus, $y$ values in the interval $(-0.1,0.1)$ represent survivors $(0$ with added jitter), and y values in the interval $(0.9,1.1)$ represent non-survivors ( 1 with added jitter)

$1.63-3.75, p<0.001)$ in the cardiac arrest subgroup, and 5.53 (95\% CI 2.82-12.62, $p<0.001$ ) in the trauma subgroup.

The AUCs for the logistic regression models on 30-day mortality for penKid was 0.71 (95\% CI 0.68-0.73) in the ICU population, 0.61 (95\% CI 0.56-0.66) in sepsis, 0.73 (95\% CI 0.66-0.80) in cardiac arrest, and 0.84 (95\% CI 0.76-0.92) in trauma. See Fig. 2.

In the ICU population, penKid had a positive association with day-two renal SOFA as well as with cardiovascular, hepatic, and neurological day-two SOFA subscores as presented in Table 2 and Fig. 3. Associations between penKid levels and day-two SOFA subscores in the sepsis, cardiac arrest and trauma subgroups are presented in Table 2.

\section{SAPS -3 adjusted models}

Thirty-day mortality was higher for high penKid levels, independently of SAPS-3, with an OR of 1.32 (95\% CI 1.17-1.50, $p<0.001$ ) in the entire ICU population, 1.76 (95\% CI $1.17-2.73, \mathrm{p}=0.0082)$ in the cardiac arrest subgroup and 3.64 (95\% CI 1.78-8.51, p = $0.0011)$ in the trauma subgroup. For sepsis, there was no clear association (OR 1.13, 95\% CI 0.94-1.36, $\mathrm{p}=0.20$ ).

The AUCs for the logistic regression models on 30-day mortality for the ICU population using SAPS-3 was 0.81 (95\% CI 0.79-0.83) versus 0.82 (95\% CI 0.79-0.84) 


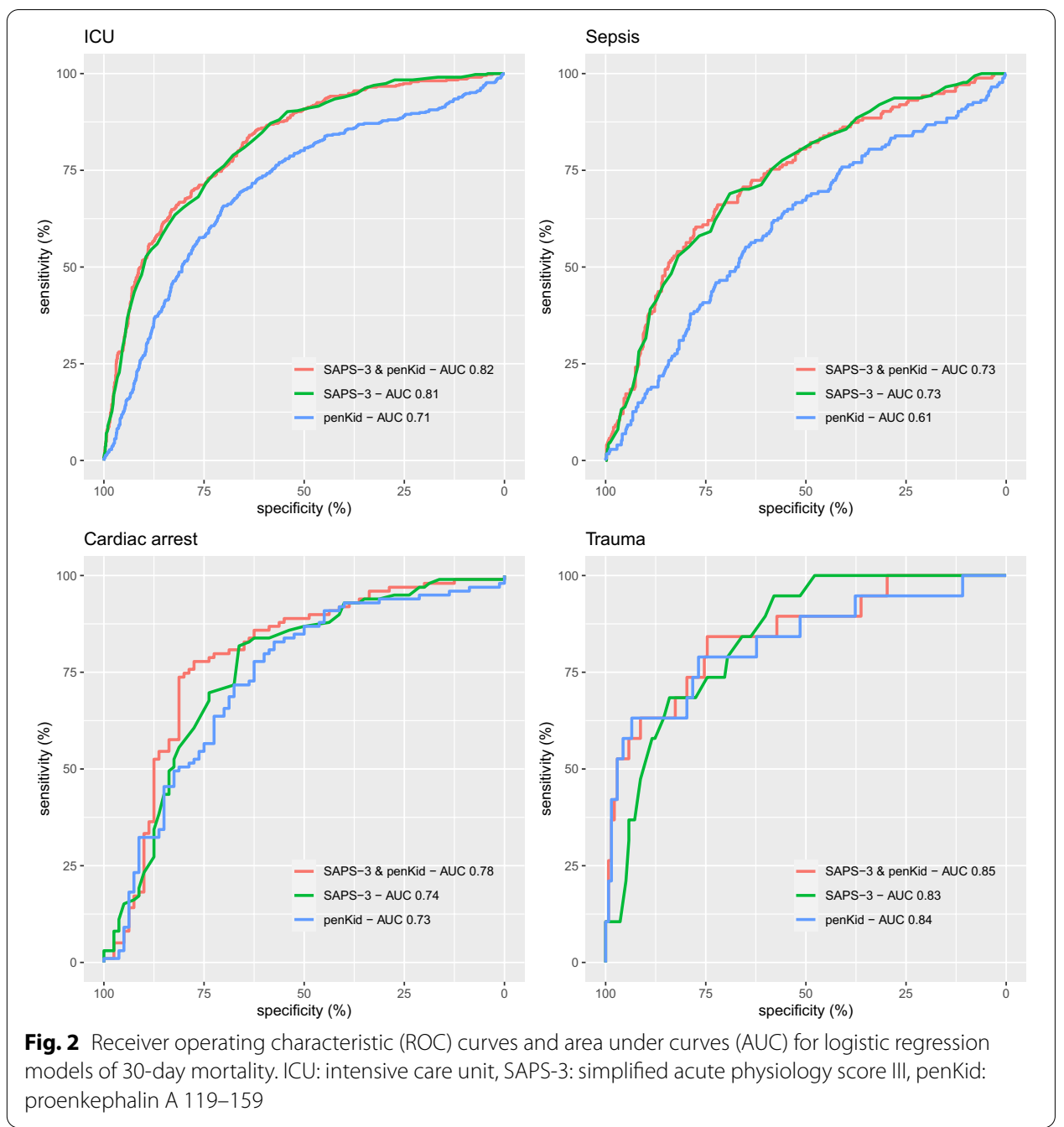

using SAPS-3 and penKid $(\mathrm{p}=0.22)$. For the sepsis population, the AUC was 0.73 (95\% CI 0.69-0.77) using SAPS-3 versus 0.73 (95\% CI 0.69-0.77) using SAPS-3 and penKid $(\mathrm{p}=0.62)$. For the cardiac arrest population, the AUC was $0.74(95 \% \mathrm{CI}$ $0.67-0.82)$ using SAPS-3 versus 0.78 (95\% CI 0.71-0.85) using SAPS-3 and penKid $(\mathrm{p}=0.018)$. For the trauma population, the AUC was 0.84 (95\% CI 0.76-0.92) using SAPS-3 versus 0.85 (95\% CI $0.75-0.95)$ using SAPS-3 and penKid $(\mathrm{p}=0.44)$. See Fig. 3.

\section{Admission creatinine adjusted models}

After correction for admission creatinine, penKid levels were positively associated with cardiovascular and neurological day-two SOFA subscores in the entire ICU population and the sepsis population as presented in Fig. 3 and in Table 2. In the cardiac arrest and the trauma subgroups, there was an association $(\mathrm{OR}>1.9)$ with neurological day-two SOFA. See Table 2. 


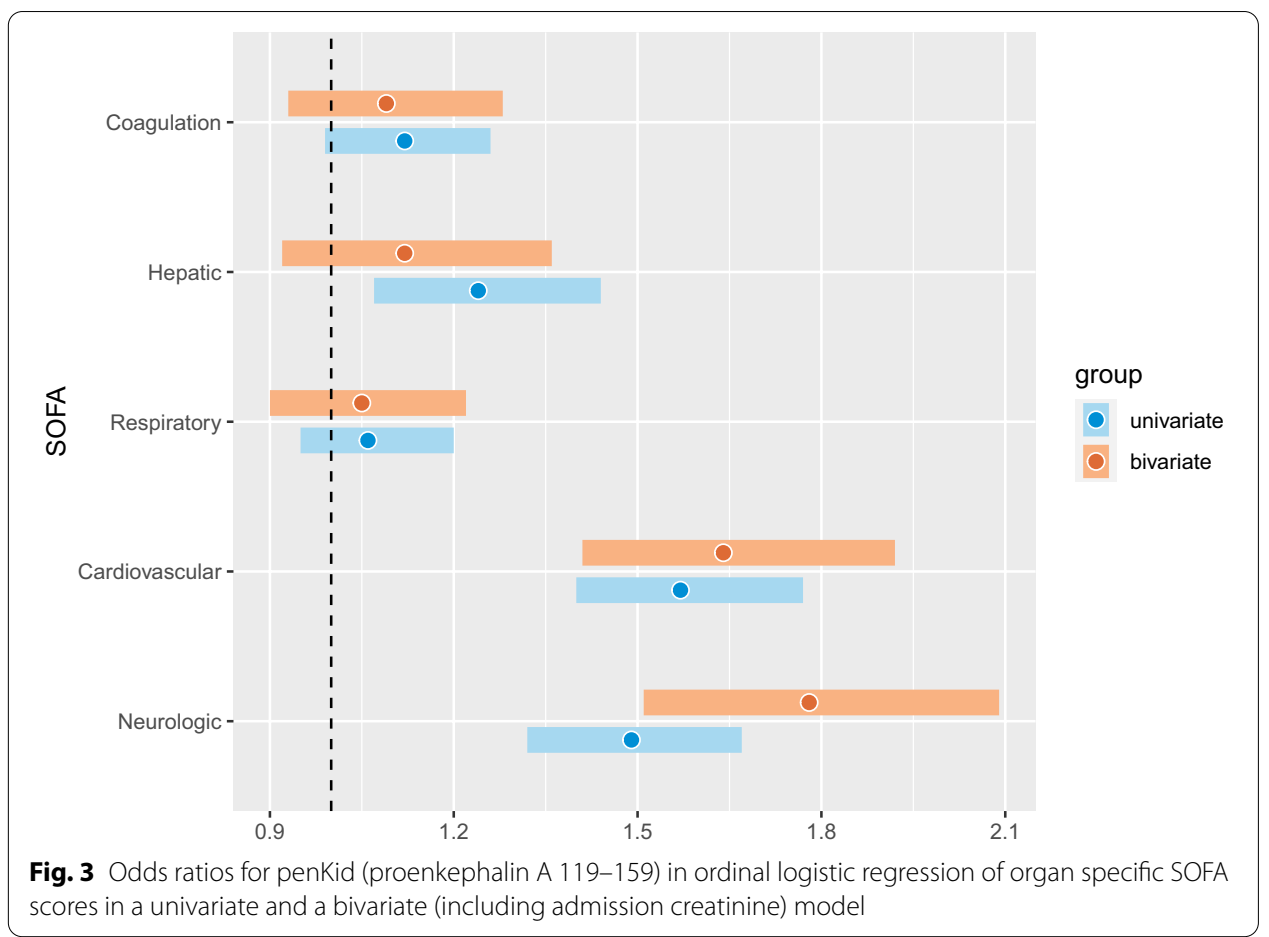

\section{Prediction of renal failure and dialysis}

The AUC for the logistic regression models on day-two renal dysfunction using admission plasma creatinine as a predictor was 0.91 versus 0.86 using penKid only $(\mathrm{p}<0.001)$. Combining admission plasma creatinine with penKid did not improve the prognostication of day-two renal failure ( 0.91 vs. $0.86, \mathrm{p}=0.54$ ). The AUC for the logistic regression models on dialysis use for the ICU population using admission plasma creatinine as a predictor was 0.88 versus 0.81 using penKid only $(p<0.001)$. Combining admission plasma creatinine with penKid did not improve the prognostication of dialysis use $(0.85$ vs. $0.88, \mathrm{p}=1)$.

\section{Discussion}

Elevated plasma penKid was prognostic of mortality in the entire ICU population, and in all the subgroups. It performed best in the trauma subgroup. In cardiac arrest patients, a combination of penKid and SAPS-3 resulted in significantly better prediction of mortality than SAPS-3 alone.

Several clinical studies have shown that penKid levels correlate with renal function as measured by plasma creatinine or an AKI definition [24]. Our findings corroborate these results by showing that penKid is associated with day-two renal dysfunction as measured by renal SOFA.

In addition, plasma penKid was positively associated with 30-day mortality and daytwo cardiovascular, hepatic, and neurological dysfunction as measured by SOFA. To explore the cause of penKid elevation from organ systems other than renal dysfunction in our ICU cohort, we corrected for admission creatinine. This removed a reno-hepatic dysfunction, which led us to hypothesise that these two organ systems were both failing 
due to a common pathway also leading to penKid elevation. What remained was a component of cardiovascular dysfunction coupled with elevated penKid only seen in the sepsis group, and a third distinct pathway of neurological dysfunction coupled with penKid elevation observable in the entire ICU population and all the subgroups. To summarise, we argue that there is an obvious renal dysfunction component to penKid elevation, a cardiovascular dysfunction component mainly in sepsis, and a neurologic dysfunction component in the entire ICU population. The association between neurologic dysfunction and elevated penKid is intriguing and we propose evaluation of penKid as a potential marker of brain injury. In further studies we aim to analyse the association of penKid with known brain injury biomarkers such as neuron-specific enolase (NSE), and serum neurofilament light (NFL) in cardiac arrest patients [25, 26]. In addition, we aim to further investigate penKid in the subgroup of trauma patients suffering from traumatic brain injury.

In relation to our brain-injury hypothesis, we suggest that a plausible explanation why elevated penKid in patients not meeting the AKI criteria ("subclinical AKI") was shown to predict mortality after adjustments in the study by Deprét et al. [8], might be due to a degree of brain injury in those patients (of which $4.4 \%$ had suffered trauma and $8.8 \%$ had suffered out-of-hospital cardiac arrest, i.e. similar to our cohort).

A strength of our study is the well-defined subgroups. The sepsis subgroup, which is often difficult to define, has been carefully characterised and described recently [18]. The cardiac arrest and trauma subgroups are common diagnoses in the ICU and are generally easily identified. Another strength is that we have adjusted for age, comorbidities, and acute severity of illness through the validated SAPS-3 score. After adjustments, our results still hold, showing that penKid provides clinically important information not captured by the SAPS-3 determinants.

\section{Limitations}

The lack of baseline creatinine levels in our study population prevented classification of AKI according to the Kidney Disease Improving Global Outcomes (KDIGO) criteria [27].

The day-two neurological SOFA score for cardiac arrest patients is determined by the fact that most patients are sedated for temperature control on day two [28], and this is therefore not an optimal measure of neurological prognosis.

It should be pointed out that our study was not specifically aimed at investigating the cardiac arrest subgroup and did therefore not correct for well-known cardiac arrest-specific prognostic variables [29].

\section{Interpretation}

To our knowledge, this is the first study investigating the value of admission penKid in a general ICU population as an independent mortality predictor and subsequent organdysfunction. Our findings open up a new path of risk assessment for critically ill patients.

\section{Generalisability}

The multicenter approach, with patients from both large university hospital ICUs and regional ICUs, make the results applicable to other high-income hospital settings. 


\section{Conclusion}

Plasma penKid on ICU admission is associated with day-two organ dysfunction and predictive of 30-day mortality in a mixed ICU-population, as well as in sepsis, cardiac arrest and trauma subgroups.

\section{Abbreviations}

penKid: Proenkephalin A 119-159; ICU: Intensive care unit; AUC: Area under the receiver operating characteristic curve; AKI: Acute kidney injury; SOFA: Sequential organ failure assessment; SAPS-3: Simplified acute physiology score III; EDTA: Ethylenediaminetetraacetic acid-plasma; IQR: Interquartile range; OR: Odds ratio; GFR: Glomerular filtration rate; TBI: Traumatic brain injury.

\section{Supplementary Information}

The online version contains supplementary material available at https://doi.org/10.1186/s40635-021-00396-6.

Additional file 1. Additional tables and figures.

\section{Acknowledgements}

We would like to thank all staff at the ICUs of Skåne University Hospital in Malmö and Lund, Helsingborg Hospital, and Kristianstad hospital for their contribution to this study. We also extend special thanks to Professor Peter Nilsson of Lund University. This study would not have been possible without the kind contribution of SphingoTec GmbH, who analysed our blood samples free of charge.

\section{Authors' contributions}

AF and HF designed the study. HL designed the biobank and compiled the data set. ML, OL, MS, MA collected clinical data. AF and ML designed the clinical database. AF performed the statistical analysis and prepared the figures. AF and LB wrote the initial manuscript. All authors read and approved the final manuscript.

\section{Funding}

Open access funding provided by Lund University. Regional research support, Region Skåne; Government funding of clinical research within the Swedish National Health Services (ALF) 2019:YF0053; Biobanking and BioMolecular resources Research Infrastructure (BBMRI, Sweden); Hans-Gabriel and Alice Trolle-Wachtmeister Foundation for Medical Research; European Union Interreg programme IV A; SphingoTec GmbH, Hennigsdorf, Germany, provided all analyses of all samples. The funding organisations had no role in the design and conduct of the study; collection, management, analysis, and interpretation of the data; preparation, review, or approval of the manuscript; or decision to submit the manuscript for publication.

\section{Availability of data and materials}

The datasets used during the current study are available from the corresponding author on request.

\section{Declarations}

\section{Ethical approval and consent to participate}

Ethical approval was obtained from the Regional Ethical Review Board of Lund, Sweden (registration nos. 2015/267 and 2017/802). Patients and their next-of-kin were given the possibility to opt out.

\section{Competing interests}

The authors declare that they have no competing interests. Proenkephalin A 119-159 was analysed free of charge by SphingoTec GmbH, Neuendorfstraße 15A, 16761 Hennigsdorf, Germany. The authors have no financial or any other interests in SphingoTec and have not been in any way influenced by the company in writing this research paper.

\section{Author details}

${ }^{1}$ Department of Clinical Sciences, Anaesthesiology and Intensive Care, Lund University, 22185 Lund, Sweden. ${ }^{2}$ Skåne University Hospital, Intensive and Perioperative Care, 22185 Lund, Sweden. ${ }^{3}$ Skåne University Hospital, Intensive and Perioperative Care, 21428 Malmö, Sweden. ${ }^{4}$ Kristianstad Central Hospital, Anaesthesia and Intensive Care, 29185 Kristianstad, Sweden. ${ }^{5}$ Helsingborg Hospital, Anaesthesia and Intensive Care, 25187 Helsingborg, Sweden. ${ }^{6}$ Skåne University Hospital, Department of Neurology, 22185 Lund, Sweden. ${ }^{7}$ Skåne University Hospital, Research and Education, 22185 Lund, Sweden.

Received: 18 February 2021 Accepted: 21 May 2021

Published online: 19 July 2021 


\section{References}

1. Metnitz PG, Moreno RP, Almeida E, Jordan B, Bauer P, Campos RA et al (2005) SAPS 3-From evaluation of the patient to evaluation of the intensive care unit. Part 1: Objectives, methods and cohort description. Intensive Care Med 31(10):1336-44

2. Moreno RP, Metnitz PG, Almeida E, Jordan B, Bauer P, Campos RA et al (2005) SAPS 3-From evaluation of the patient to evaluation of the intensive care unit. Part 2: Development of a prognostic model for hospital mortality at ICU admission. Intensive Care Med 31(10):1345-55

3. Ernst A, Köhrle J, Bergmann A (2006) Proenkephalin A 119-159, a stable proenkephalin A precursor fragment identified in human circulation. Peptides. 27(7):1835-1840

4. Denning GM, Ackermann LW, Barna TJ, Armstrong JG, Stoll LL, Weintraub NL et al (2008) Proenkephalin expression and enkephalin release are widely observed in non-neuronal tissues. Peptides. 29(1):83-92

5. Donato LJ, Meeusen JW, Lieske JC, Bergmann D, Sparwaßer A, Jaffe AS (2018) Analytical performance of an immunoassay to measure proenkephalin. Clin Biochem. 58:72-77

6. Beunders R, van Groenendael R, Leijte GP, Kox M, Pickkers P (2020) Proenkephalin compared to conventional methods to assess kidney function in critically III sepsis patients. Shock. 2020;54(3):308-314

7. Hollinger A, Wittebole X, François B, Pickkers P, Antonelli M, Gayat E et al (2018) Proenkephalin A 119-159 (Penkid) is an early biomarker of septic acute kidney injury: the kidney in sepsis and septic shock (Kid-SSS) study. Kidney Int Reports. 3(6):1424-1433

8. Dépret F, Hollinger A, Cariou A, Deye N, Vieillard-Baron A, Fournier MC, et al (2020) Incidence and outcome of subclinical acute kidney injury using penkid in critically III patients. Am J Respir Crit Care Med. 2020 09;202(6):822-829

9. Stark M, Danielsson O, Griffiths WJ, Jörnvall H, Johansson J (2001) Peptide repertoire of human cerebrospinal fluid: novel proteolytic fragments of neuroendocrine proteins. J Chromatogr B. 754(2):357-367

10. Marino R, Struck J, Hartmann O, Maisel AS, Rehfeldt M, Magrini L et al (2015) Diagnostic and short-term prognostic utility of plasma pro-enkephalin (pro-ENK) for acute kidney injury in patients admitted with sepsis in the emergency department. J Nephrol. 28(6):717-724

11. Kim H, Hur M, Lee S, Marino R, Magrini L, Cardelli P et al (2017) Proenkephalin, neutrophil gelatinase-associated lipocalin, and estimated glomerular filtration rates in patients with sepsis. Ann Lab Med. 37(5):388-397

12. Yalcin A, Baydin A, Tuncel ÖK, Erenler AK, Çokluk C, Güzel M et al (2017) Diagnostic values of proenkephalin and S100B protein in traumatic brain injury. LaboratoriumsMedizin. 41(3):123-128

13. Gao JB, Tang WD, Wang X, Shen J (2014) Prognostic value of neuropeptide proenkephalin A in patients with severe traumatic brain injury. Peptides. 58:42-46

14. Doehner W, von Haehling S, Suhr J, Ebner N, Schuster A, Nagel E et al (2012) Elevated plasma levels of neuropeptide proenkephalin A predict mortality and functional outcome in ischemic stroke. J Am College Cardiol. 60(4):346-354

15. Chen XL, Yu BJ, Chen MH (2014) Circulating levels of neuropeptide proenkephalin A predict outcome in patients with aneurysmal subarachnoid hemorrhage. Peptides. 56:111-115

16. Yang XG, An HL, Zhang JM (2014) Neuropeptide proenkephalin A is associated with in-hospital mortality in patients with acute intracerebral hemorrhage. Peptides. 58:47-51

17. Bossuyt PM, Reitsma JB, Bruns DE, Gatsonis CA, Glasziou PP, Irwig L et al (2015) STARD 2015: an updated list of essential items for reporting diagnostic accuracy studies. BMJ. $351:$ h5527

18. Lengquist M, Lundberg OHM, Spångfors M, Annborn M, Levin H, Friberg H, et al (2020) Sepsis is underreported in Swedish intensive care units: a retrospective observational multicentre study. Acta Anaesthesiol Scand. 2020;64(8):1167-1176

19. Ferreira FL, Bota DP, Bross A, Mélot C, Vincent $J$ (2001) Serial evaluation of the SOFA score to predict outcome in critically ill patients. JAMA. 286(14):1754-1758

20. Hosmer DW, Lemeshow S (2000) Applied logistic regression. Wiley series in probability and mathematical statistics. New York: Wiley

21. Hothorn T, Möst L, BühImann P (2018) Most likely transformations. Scandinavian J Stat. 45(1):110-134

22. Fawcett T (2015) An introduction to ROC analysis. Pattern Recogn Lett 27(8):861-874

23. DeLong ER, DeLong DM, Clarke-Pearson DL (2018) Comparing the Areas under two or more correlated receiver operating characteristic curves: a nonparametric approach. Biometrics 3:837

24. Khorashadi M, Beunders R, Pickkers P, Legrand M (2020) Proenkephalin: a new biomarker for glomerular filtration rate and acute kidney injury. Nephron. 2020; p. 1-7

25. Freeman WD, Chiota NA (2011) Neuron-specific enolase correlates with other prognostic markers after cardiac arrest. Neurology. 77(20):1856-1857

26. Moseby-Knappe M, Mattsson N, Nielsen N, Zetterberg H, Blennow K, Dankiewicz J, et al (2019) Serum neurofilament light chain for prognosis of outcome after cardiac arrest. JAMA Neurol. 2019 01;76(1):64-71

27. Khwaja A (2012) KDIGO clinical practice guidelines for acute kidney injury. Nephron Clin Pract. 120(4):C179-c184

28. Nolan JP, Soar J, Cariou A, Cronberg T, Moulaert VR, Deakin CD et al (2015) European Resuscitation Council and European Society of Intensive Care Medicine Guidelines for Post-resuscitation Care 2015: Section 5 of the European Resuscitation Council Guidelines for Resuscitation 2015. Resuscitation. 95:202-222

29. Søholm H, Hassager C, Lippert F, Winther-Jensen M, Thomsen JH, Friberg H et al (2015) Factors associated with successful resuscitation after out-of-hospital cardiac arrest and temporal trends in survival and comorbidity. Ann Emerg Med. 65(5):523-531

\section{Publisher's Note}

Springer Nature remains neutral with regard to jurisdictional claims in published maps and institutional affiliations. 